\title{
Ultra-short periods of wakeful rest promote the viewpoint-invariance of entities mnemonic representations
}

\author{
Pierre-Yves Jonin ${ }^{1}$, Julie Coloigner ${ }^{1}$, Elise Bannier ${ }^{1}$, Christian Barillot ${ }^{1}$, Gabriel Besson ${ }^{2}$ \\ ${ }^{1}$ University of Rennes I, CNRS, Inria, Inserm, IRISA UMR 6074, Empenn Team - ERL \\ U1228, Rennes, France. \\ ${ }^{2}$ GIGA-CRC In-Vivo Imaging, University of Liège, Belgium.
}

\begin{abstract}
Humans can recognize thousands of visual objects after a single exposure, even against highly confusable objects, and despite viewpoint changes between learning and recognition. Memory consolidation processes like those taking place during wakeful rest contribute to such a feat, possibly by protecting the fine details of objects' representations. However, whether rest-related consolidation promotes the viewpoint invariance of mnemonic representations for individual objects remains unexplored.

Fifteen participants underwent a speeded visual recognition memory task tapping on familiarity-based recognition of individual objects, across four conditions manipulating postencoding rest. Viewpoints of target items were modified between study and test while controlling study-test perceptual distance, and targets and lures shared the same subordinate category, making recognition independent from perceptual and conceptual fluency. Performance was very accurate, even without post-encoding rest, which did not enhance memory. However, rest uniquely made target detection immune to study-test perceptual distance.

These findings suggest that very short periods of wakeful rest (down to 2-sec post-stimulus) suffice to achieve complete mnemonic viewpoint-invariance, pushing forward the strength of post-encoding rest in learning and memory. They also strongly argue for a holistic, viewpointinvariant, mnemonic representation of visual objects.
\end{abstract}




\section{Introduction}

Imagine having to pick up your coat before you leave a party. Even if a coat identical to yours hangs next to it on the coat rack, it doesn't prevent you from picking up the right one. Many situations require our visual memory system to allow recognition of a unique item, despite 'ambiguity', resulting from context-dependent changes (light, orientation, contrast, view angle, etc.). We excel at recognizing visual objects as specific 'entities' distinguished from any other exemplar of the same subcategory, with an impressive storage capacity and even after a single exposure ${ }^{1,2}$, despite major changes in visual presentation ${ }^{3}$ (e.g. across viewpoints) and in a fraction of a second ${ }^{4}$. Yet, how can novel individual visual objects be memorized after only a single or few exposures, so that they can then be recognized so easily and quickly despite changes in their presentation remains largely elusive.

Such ease and speed indicate how such retrieval may rely on familiarity (automatic and fast), more than on recollection (much slower and controlled) ${ }^{5-7}$. Importantly, the mnemonic representations that will subsequently support such a 'familiarity for individual visual objects' need to withstand long-term delays. Consolidation - allowing representations to be transformed into memories remaining accessible seconds, minutes or years after encoding ${ }^{8}-$ is thereby critical. Before sleep consolidation (well-known to benefit memory ${ }^{9}$ ), fast offline consolidation processes already take place during brief post-encoding awake periods characterized by minimal sensory input ${ }^{10,11}$.

While post-encoding wakeful rest enhances verbal and visual memory ${ }^{12-21}$, only hippocampal-dependent memories, i.e. relational memories or memories for the event ${ }^{22,23}$ (including the -temporal and/or spatial- flexible binding of the object(s) and contextual features) have been under scrutiny so far. In fact, prior research reporting rest-related memory enhancement, including those focusing on visual objects mnemonic representations ${ }^{15}$, only used tasks primarily relying on recollection (e.g. free recall, unspeeded recognition memory, or using novel associations as material), known to depend on slow and controlled processes involving hippocampal mnemonic representations ${ }^{5,24}$. Yet, mnemonic representations of individual visual objects, subsequently accessible easily and quickly on the basis of familiarity, are thought to rely on non-hippocampal computations ${ }^{25}$. As a consequence, we largely ignore whether and how wakeful rest contributes to fast familiarity for individual visual objects, which may nevertheless constitute most of our everyday mnemonic decisions. The present study aims at filling this gap.

Importantly, several levels of mnemonic representations could support familiarity. Familiarity would arise from fluency (the increased ease with which processing re-occurs) when it is heuristically attributed to previous experience ${ }^{26}$. Each level of visual processing along the representational hierarchy is independently susceptible to occur more fluently at the 
re-exposure of an item (e.g. ${ }^{27}$ ), such that when studying the effect of wakeful rest on fast familiarity, it appears critical to avoid confounding them. Conceptual and perceptual fluency are two well-defined sources of familiarity ${ }^{28}$. However, none of them appears sufficient to achieve accurate fast familiarity for individual visual objects. Indeed, as defined above, such a familiarity is able to discriminate two individual exemplars from the same subcategory which hence present similar conceptual fluency, so that it cannot rely on conceptual fluency per se. Likewise, familiarity for individual visual objects cannot rely only on perceptual fluency either, since it allows accurate detection of an individual visual object despite changes in its visual presentation. Growing evidence points to a specific individual-level processing of visual objects, representing them holistically and viewpoint-invariantly ${ }^{29,30}$. At re-exposure of a given visual object, processing at this level could thus also show enhanced fluency, thereby constituting another independent and even more relevant (albeit mostly overlooked) source of familiarity ${ }^{31}$. However, whether rest-related consolidation is necessary for individual-level familiarity has never been studied. Here, we thus designed a familiarity task controlling for the contribution of both conceptual and perceptual fluency to assess the effect of wakeful rest on familiarity for individual visual objects specifically relying on individual-level mnemonic representations.

First, after an explicit learning (study) phase, followed by a 2-minutes break (Fig. 1A), this study aimed at directly assessing fast familiarity for individual visual objects using the Speed and Accuracy Boosting procedure (SAB) ${ }^{32,33}$, which constrains participants to recognize Old items (targets) using their fastest strategy, namely familiarity, thus minimizing the contribution of recollection. Briefly, this Go/No-Go speeded procedure simply requires go-responses for targets to be given before $600 \mathrm{~ms}$, indicating success or failure by a brief audio feedback. In order to prevent respectively conceptual and perceptual fluency to contribute to familiarity, New items (lures) were drawn from the same subordinate semantic category as targets, and target stimuli were not presented at test as during the study phase (size, luminance, contrast and in-plane rotation were pseudo-randomly modified). Thereby, neither conceptual nor perceptual fluency could be decisive for accurate recognition. Specifically, to control perceptual fluency even more precisely, the study-test perceptual distance (computed based on the bio-inspired Hmax algorithm ${ }^{34}$ ) between studied items and their corresponding target items used at test was matched to the study-test perceptual distance between studied items and their corresponding lures used at test (Fig. 1C). By assessing in each participant the relationship between study-test perceptual distance and accuracy across items, it was thus possible to quantify the sensitivity of accuracy to study-test perceptual changes. Second, this study examined the contribution of wakeful rest using 4 experimental conditions in a withinsubjects design, that differed depending on whether and when awake rest was allowed (Fig. 1B). In the 'no rest' (NR) condition, no cognitive rest was allowed either at study (i.e. after 
each stimulus presentation, by using brief Inter-Stimulus-Intervals (ISI) of 100-200 ms), or in the delay phase (i.e. the 2-minutes delay was filled with an imagery navigational task where participants had to describe out loud each and every landmark they saw and its position, while mentally navigating between two locations randomly chosen by the experimenter, e.g. 'your place to the university'). This NR condition served as the baseline condition, identical to all three other conditions in all aspects but one. Cognitive rest was allowed only at study in the 'study rest' (SR) condition (i.e. ISIs were jittered in the 2000-3000 ms range), and only during the delay phase in the 'delay rest' (DR) condition (i.e. the 2-minutes delay was filled with an openeyed rest period where participants were instructed to relax, letting their mind wander and avoiding to focus on any thought). Finally, the 'no rest unspeeded' (NRU) condition was identical to the NR condition except for the testing phase that was unspeeded.

In the current study, we thus addressed whether and how rest-related consolidation contributes to familiarity for individual visual objects, by probing the effect of brief wakeful rest periods on a task specifically tapping on individual-level familiarity for naturalistic visual objects. Hypothesizing the existence of a specific individual level of representation, we predicted that even the NR condition would be successfully performed. In other words, such a level of mnemonic representations should allow accurate familiarity-based recognition even when mnemonic representations at lower, conceptual and perceptual levels are irrelevant for the task and no wakeful rest is allowed. Importantly though, prior research has suggested that rest-related consolidation would be involved in either generalization processes ${ }^{35,36}$ or in protecting the fine details of newly formed memories by enhancing the fine-grained representations of visual objects ${ }^{15}$. Hence, extending these reports - mainly tapping on recollection-based recognition - to familiarity for individual visual objects, leaves us with two hypotheses. Wakeful rest may either contribute to the generalization of mnemonic representations across viewpoints, or promote lure discrimination through the enhancement of the fine resolution of mnemonic representations.

We reasoned that these hypotheses make non overlapping predictions with respect to individual-level familiarity (i.e. speeded recognition conditions, 'NR', 'SR' \& 'DR'). If the 'resolution' hypothesis holds, then wakeful rest should promote lure discrimination (because of enhanced consolidation of the fine details of mnemonic representations) and thus lower the false alarm rates. Meanwhile, the hit rates should remain unaffected, thus resulting in increased accuracy. Moreover, wakeful rest should make the false alarm rates independent from perceptual distances between lures and studied targets. Indeed, if rest protects the fine details of visual objects' memories, whether the lures are perceptually similar or dissimilar to targets should not affect the availability of high-resolution targets representations to provide an accurate 'New' response. If the 'generalization' view holds however, then wakeful rest should increase the hit rates due to better generalization of mnemonic representations across 
viewpoints, leaving unaffected the false alarm rates, thus resulting in increased accuracy. Moreover, wakeful rest, by making mnemonic representations viewpoint-invariant, should also make the hit rates independent from study-test perceptual distances. However, with respect to unspeeded recognition memory (i.e. 'NRU' condition), letting recollection contribute while preventing wakeful rest should result in increased accuracy overall, since recollection is more accurate than familiarity and that speed imposes a trade-off with accuracy ${ }^{24}$. Furthermore, given that slow, controlled retrieval processes associated with recollection can rely on either item-related or contextual cues, no specific prediction could be made in that condition regarding the relationship between accuracy and perceptual distance.

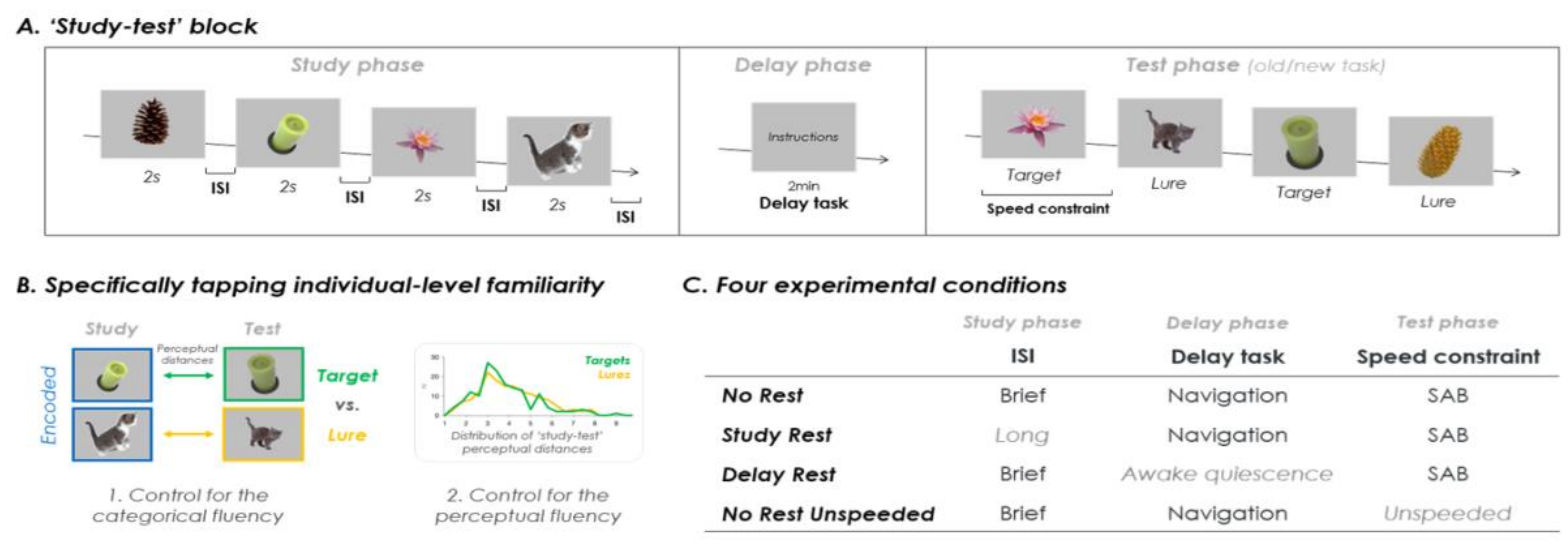

Figure 1. Experimental design. A. 'Study-test' blocks are composed of three phases: a study phase, during which pictures of objects $(\mathrm{N}=26)$ are presented (twice in the phase) during 2 s separated by an inter-stimulus interval (ISI) long or brief depending on the condition (B); a delay phase, during which participants undergo a 2-min delay task depending on the condition (B); a test phase, during which participants need to discriminate studied object exemplars (targets) despite random change in their presentation (i.e. in size, luminance, contrast and in-plane orientation), against novel object exemplars (lures) despite being drawn from studied subordinate categories, under speed constraints (depending on the condition). B. To specifically assess familiarity for unique objects exemplars, perceptualconceptual sources of familiarity, i.e. categorical and low-level fluency, were controlled by ensuring that the subordinate category of a tested stimuli as well as its perceptual distance with its encoded version could not help its discrimination as being a target or a lure. C. Across the four experimental conditions, wakeful rest was manipulated as shown in the table (see text for detailed timing). 


\section{Results}

\section{Accurate visual object familiarity without diagnostic cues from perceptual or conceptual fluency}

Participants succeeded very well across all 4 experimental conditions ('No Rest': accuracy $(A z)=.84$ (.06), 95\% Cl [.82,.87], 'Study Rest': $A z=.85$ (.05) [.82,.87], 'Delay Rest': $A z=.86$ (.06) $[.83, .88]$, 'No Rest Unspeeded': $A z=.93$ (.04) [.91,.95]; GLMM's intercept effect on accuracy $(A z)$ - i.e. reference condition 'No Rest' against 0 : $F(1,56)=2880, p<1$ e-49; see Figure 2), showing that, even in the absence of cognitive rest between study and test, and despite the irrelevance of perceptual and conceptual fluency, mnemonic representations for unique individual objects could be formed after as few as two exposures, and further retrieved easily and quickly from long-term memory on the basis of familiarity.

\section{No benefit of post-encoding wakeful rest on accuracy of visual object familiarity}

The GLMM for accuracy further revealed a main effect of the condition $(F(3,56)=12.7, p<$ 1e-05). Yet, compared to the reference 'No Rest' condition, only 'No Rest Unspeeded' was better succeeded ( $\beta=0.0883$ (0.0168) [0.0547,0.122], $t(56)=5.27, p<1 \mathrm{e}-05$ ), while neither the 'Study Rest' ( $\beta=0.00374$ (0.0121) [-0.0206,0.028], $t(56)=0.308, p=.759$ ) nor the 'Delay Rest' $(B=0.0175(0.015)[-0.0126,0.0475], t(56)=1.16, p=.250)$ showed any difference, evidencing the typical benefit of recollection availability on recognition memory, but no effect of the cognitive rest manipulations on global accuracy, contrary to our hypotheses.

The GLMM on the bias measure $\left(B^{\prime \prime}\right)$ revealed a main effect of the condition $(F(3,56)=25.5$, $p<1 \mathrm{e}-9)$. Compared to the reference 'No Rest' condition, only 'No Rest Unspeeded' showed a different bias ('No Rest': $B$ "' = .45 (.04) [.43,.47]; 'No Rest Unspeeded': B'= .57 (.06) [.55,.60]; $B=0.125$ (0.0147) $[0.0959,0.155], t(56)=8.54, p<1 \mathrm{e}-10)$, while neither the 'Study Rest' ( $B$ " $=.46(.04)[.45, .48] ; \beta=0.0152(0.0106)[-0.00597,0.0363], t(56)=1.44, p=.156)$ nor the 'Delay Rest' $(B$ ' = .44 (.04) $[.42, .46] ; \beta=-0.00442(0.00917)[-0.0228,0.014], t(56)=-0.482$, $p=.632$ ) showed any difference. Overall, biases were conservative (i.e. reference condition 'No Rest' against 0: $F(1,56)=2170, p<1 \mathrm{e}-45, \beta=0.448$ (0.00962) $[0.429,0.468])$, exception made of the 'No Rest Unspeeded' where they were liberal.

Considering targets only, the GLMM on hit rates showed a main condition effect $(F(3,56)=$ 49.5, $p<1 \mathrm{e}-15$ ) with, compared to the 'No Rest' condition (hit rate $(\%)=70.94(6.97)$ [67.86,74.36]), only 'No Rest Unspeeded' showing a better hit rate ( hit rate $=91.45(5.45)$ [88.21,93.50], $B=0.205$ (0.0191) [0.167,0.243], $t(56)=-4.61, p<1 e-14)$, while neither the 'Study Rest' ( hit rate $=72.99$ (6.71) [69.91,76.41], $B=0.0205(0.0188)[-0.0171,0.0581], t(56)$ 
$=1.09, p=.279)$ nor the 'Delay Rest' (hit rate $=72.14(6.56)[69.57,75.38], \beta=0.012(0.0164)$ $[-0.0209,0.0448], t(56)=0.73, p=.468)$ showed any difference. The absence of rest-related benefit for target detection thus seemed to favor the 'resolution' hypothesis (see Introduction).

Considering lures only however, the GLMM on false alarm rates did not show any main effect of the condition $(F(3,56)=1.45, p=.237$; 'No Rest': false alarm rate $(\%)=17.95(6.99)$ [15.21,20.17], 'Study Rest': false alarm rate = 19.15 (6.77) [16.41,22.22], 'Delay Rest': false alarm rate $=16.24(6.83)[12.99,19.49]$, 'No Rest Unspeeded': false alarm rate $=20.85(9.88)$ $[16.92,26.50])$, suggesting that the pattern of effects observed on global accuracy was mainly driven by hits (i.e. more in the 'No Rest Unspeeded' condition, when recollection was available). Here, the absence of effect of the conditions on lure discrimination therefore spoke for the 'generalization' hypothesis put forward in the introduction.

\section{Post-encoding wakeful rest improves viewpoint-invariance of visual object familiarity}

To assess whether wakeful rest could promote the viewpoint invariance of accurate visual familiarity for objects, we tested the relationship between study-test perceptual distance and the rate of correct responses to targets or the rate of incorrect responses to lures (Hits and FAs, respectively, see Methods). We found that, only in the 'No Rest' and 'No Rest Unspeeded' conditions, the hit rates were sensitive to study-test perceptual changes (No Rest vs. $0: t(14)=5.71, p=.024$ ), while it was not the case in the three other conditions (Study Rest vs. $0: t(14)=0.82, p=.373$, Delay Rest vs. $0: t(14)=0.05, p=.823$, No Rest Unspeeded vs. $0: t(14)=0.79, p=.382$; see Figure 2$)$. The GLMM further did not show a main effect of conditions $(F(3,56)=1.88, p=.144)$. These results suggest that wakeful rest is specifically involved in the building of a viewpoint-invariant mnemonic representation of unique individual objects.

In turn, false alarms sensitivity to study-test perceptual changes was significant in the 'No Rest' $(t(14)=17.75, p<1 \mathrm{e}-04)$, 'Study Rest' $(t(14)=6.59, p=.016)$ and 'Delay Rest' $(t(14)=$ 28.91, $p<1 \mathrm{e}-05)$ condition but not in 'No Rest Unspeeded' $(t(14)=0.73, p=.400)$. The GLMM further showed a main effect of conditions $(F(3,56)=4.14, p=.0101)$, with, compared to No Rest, No Rest Unspeeded showing a significant difference $(B=0.461(0.14)[0.18,0.742], t(56)$ $=3.28, p=.00177)$, while Study Rest $(B=0.0887(0.137)[-0.186,0.363], t(56)=0.648, p=$ $.52)$ and Delay Rest $(B=0.0428$ (0.0795) $[-0.116,0.202], t(56)=0.539, p=.592)$ did not show significant differences. These results suggest that false alarms were associated with the perceptual similarity of lure items with their paired studied items, but only to the extent that recollection-based processes are made unlikely. 
Following up on the finding that both hits and false alarms were sensitive to study-test perceptual change in the NR condition, we further tested whether one was more sensitive to perceptual change than the other. Using a two-tailed post-hoc t-test, we found that target detection was significantly less sensitive to perceptual distance than lure discrimination $(\mathrm{t}(14)$ $=2.54, p=.023)$.

A.

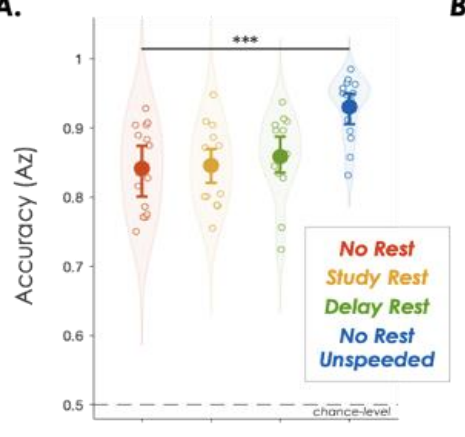

B.

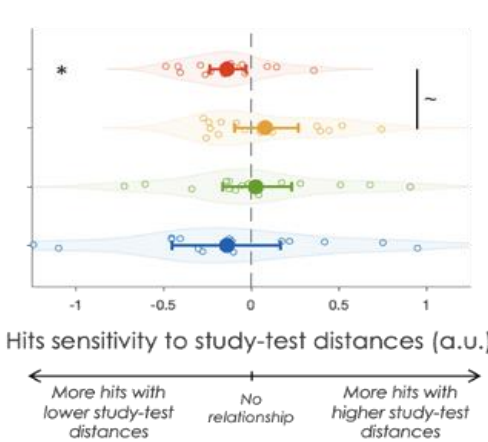

c.

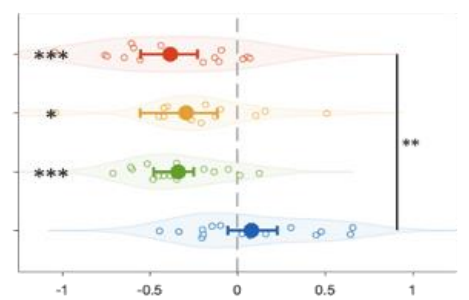

FAs sensitivity to study-test distances (a.u.)

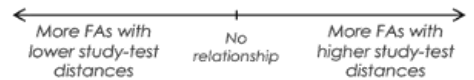

Figure 2. Scores of interest across the four conditions. A. Accuracy $(A z)$ was well above chancelevel and only dependent on the speed constraint manipulation, with only 'No Rest Unspeeded' differing from 'No Rest' condition. B. Importantly, hits were sensitive to study-test perceptual distances only in the 'No Rest' condition, but not in the 'Study Rest', 'Delay Rest' and 'No Rest Unspeeded' conditions. C. False alarms were sensitive to study-test perceptual distances in all speeded conditions (i.e. 'No Rest', 'Study Rest' and 'Delay Rest'), but not in the 'No Rest Unspeeded' condition. Overall this pattern of results suggests that short periods of unoccupied time allows for generalizing mnemonic traces beyond their viewpoint. Hits and FAs sensitivity to study-test distances ( $\mathrm{B}$ and $\mathrm{C}$ ) are expressed in the same arbitrary units (a.u.), i.e. as beta values estimated from the GLMMs (see Methods). ${ }^{* * *}: p<.001$; $*: p<.05 ; \sim: p<.08$. 


\section{Discussion}

In the current study, we addressed whether and how wakeful rest-related consolidation contributes to fast familiarity for individual visual objects. We specifically investigated the effect of wakeful rest on memory performance, and on its sensitivity to study-test perceptual distances (i.e. to viewpoint), in order to probe the 'resolution' hypothesis as well as the 'generalization' of mnemonic representations view. We found that wakeful rest did not enhance familiarity for individual visual objects, neither considering global accuracy, nor, in particular, lure discrimination (false alarm rates). However, across the two rest conditions (SR and DR), wakeful rest made target detection insensitive to changes in viewpoint, while letting unaffected the sensitivity of lure discrimination to changes in viewpoint. Importantly, we also found that accurate familiarity for individual visual objects can be achieved within $600 \mathrm{~ms}$, even when lower, perceptual and conceptual fluency signals cannot contribute to performance and no wakeful rest is allowed. Overall, we argue that these findings are in line with a role of rest-related consolidation in generalizing meaningful non-hippocampal mnemonic representations, as opposed to its role in refining resolution when it comes to event-based hippocampal mnemonic representations. Furthermore, we argue that our findings strongly speak for the existence of a mnemonic representation of individual visual objects, binding together objects' features even beyond their viewpoints to a certain degree determined by experimental conditions, making the object a unique exemplar from its category.

\section{Wakeful rest and familiarity for individual visual objects}

The first main result of this study is that familiarity for individual visual objects was not enhanced with wakeful rest being available, neither considering target detection (hit rate), nor lure discrimination (false alarm rate). This observation held for the two wakeful rest conditions, namely in the SR condition which released $\sim 2$ seconds of wakeful rest between each stimulus encoding using long ISIs compared to NR, and in the DR condition which released 2 minutes of awake quiescence in place of a resource-demanding imagery navigational task during the delay phase. This result appears at odds with studies reporting that consolidation enhances lure discrimination, but not target detection in mnemonic discrimination tasks of visual objects (namely, in versions of the well-studied Mnemonic Similarity Task, or MST, see ${ }^{15,37,38}$ ), hence leading to the idea that consolidation would enhance the fine-grained resolution of visual object memories. When compared with the unique previous study investigating this consolidation effect by means of wakeful rest ${ }^{15}$, different methodological differences could explain this discrepancy. For instance, this previous study manipulated wakeful rest between participants while our design was within-participant, controlling for a simple group effect. More 
related to consolidation itself, our periods of wakeful rest were rather brief (i.e. within-seconds between-stimuli intervals or 2-minutes post-learning delay), so that they may have been too short to benefit lure discrimination, compared to rest duration of 10 minutes (prior studies generally reported rest duration ranging from 10 to 30 minutes, but $\mathrm{see}^{39}$ ). Furthermore, perceptual fluency was controlled in our study by presenting targets differently than at study, a control that was not used before. One possibility, then, is that participants in this prior report would rely upon rest-related increased perceptual fluency to accurately identify targets. However, as stated above, lure discrimination but not target detection was reported to benefit from wakeful rest, making this factor unlikely to explain the discrepancy. Lastly, and more importantly regarding our theoretical framework, this previous study did not use speed constraints, so that performance could largely rely upon hippocampus-dependent mnemonic representations. In contrast, the use of speeded conditions (using a 600-ms response deadline) makes highly unlikely that recognition memory performance in the current study relied on hippocampal mnemonic representations, as evidenced for instance by lesion ${ }^{40}$ or intracranial electrophysiological ${ }^{41}$ human studies. While it is not possible to conclude here because of the aforementioned other methodological differences, we propose that the discrepancy observed between the present study and the prior report ${ }^{15}$ pertains to differences in the nature of the probed mnemonic representations, which wakeful rest would differentially affect. If more studies are required to test this idea, further arguments are presented along the rest of this discussion.

One critical objective of this study was to assess the generalization of mnemonic representations beyond their specific studied presentations, by investigating performance sensitivity to study-test perceptual distances, and to assess whether wakeful rest would affect it. We found that target detection was sensitive to study-test perceptual distance in the absence of wakeful rest (NR condition), but that wakeful rest (i.e. SR and DR) made it invariant to study-test perceptual distance, while letting lure discrimination at a high level of sensitivity to study-test perceptual distance. This pattern of results has several implications. First, it is noteworthy that when no wakeful rest was allowed (NR), very good performance was observed despite changes in target presentation. This suggests that some relative generalization to the studied presentation was already achieved. Yet, the fact that target detection was still sensitive to study-test perceptual distances underlines that this relative generalization was not complete in the absence of wakeful rest. Second, however, as short as two seconds between stimulus presentations at encoding (SR), or two minutes of wakeful rest in place of a navigational task during the delay phase (DR), were sufficient to make target detection fully invariant to study-test perceptual distance, suggesting that the relative level of generalization available under rest-free condition could be fully completed through the addition of ultra-short period of wakeful rest. These findings are the first to report, to the best of our 
knowledge, that ultra-short periods of wakeful rest may be sufficient to help building a mnemonic representation further accessible irrespectively of study-test perceptual changes. Third, and importantly, showing that the effect of wakeful rest on invariance to study-test perceptual distance (i.e. on viewpoint invariance) was observed for targets detection but not for lure discrimination, strongly suggests that wakeful rest specifically affects encoded individual visual objects. The effect of wakeful rest is expected to be due to consolidation, mirroring the well-known sleep-related consolidation ${ }^{42}$. Moreover, seconds-long periods of cognitive rest seem enough to promote memory formation ${ }^{39}$, a timescale compatible with our findings in the SR condition. Since only encoded mnemonic representations are expected to be affected by consolidation, the present finding that wakeful rest specifically affects encoded individual visual objects is also perfectly in line with the idea that the wakeful rest effects reported here are effectively due to consolidation. Last, this rest-related consolidation should not be understood only in terms of a mere interference prevention due to minimal sensory inputs. Indeed, by allowing objects to become recognizable beyond the specific studied presentations, rest-related consolidation also transforms the mnemonic representations. Prior research has highlighted that sleep-related consolidation not only strengthens the memory trace, but also transforms it, with, for example, sleep enhancing gist extraction (e.g. ${ }^{18,43}$ ), suggesting that consolidation processes actively alter representations, possibly contributing to concept learning ${ }^{36}$ (i.e., new explicit knowledge, for similar evidence following wakeful rest, see $\left.^{35}\right)$. Here, our view is that even short periods of wakeful rest in our experimental design were responsible for early consolidation processes, which further transforms mnemonic representations so that they become viewpoint-invariant.

\section{Three independent arguments for an individual level of visual object representations}

Of particular importance, we furthermore argue that this rest-related transformative consolidation applies here to mnemonic representations lying specifically at the putative individual-level representation, and advance three independent arguments from our findings favoring the actual existence of such an individual level of visual object representations. The first argument comes from the finding that even our most challenging condition (NR) was very successfully performed, with hit rates ranging beyond $70 \%$ and false alarm rates mostly below $30 \%$. This is remarkable per se. The Speed and Accuracy Boosting procedure - constraining participants to respond before $600 \mathrm{~ms}$ - is very challenging even for young healthy people $\mathrm{e}^{32}$. In addition, targets were expected to be harder to recognize than across prior reports, because (1) they were presented differently than during study (the study-test perceptual distance was manipulated) and (2) targets and lures were drawn from the same semantic categories. Moreover, this condition ensured that no wakeful rest-related consolidation could occur. In 
sum, this demonstrates for the first time that mnemonic representations of individual visual objects can be sufficiently consolidated from only two exposures (despite no wakeful rest permitted), and then retrieved on the basis of familiarity (while conceptual and perceptual fluency were prevented) with excellent speed and ease. We argue that this strongly favors the hypothesis of the existence of an individual level of visual object representations. Indeed, this result does not seem compatible with the proposals putting forward an independent storage of objects' features ${ }^{44,45}$, according to which visual meaningful objects would be stored as independent and hierarchically organized features, which can be retrieved - or, conversely, forgotten - independently ${ }^{46}$. From this hierarchy, in our task design, neither low-level nor category-specific features retrieval could suffice to perform accurately, since perceptual and conceptual fluency cues had no diagnostic value. Yet, targets and lures were distinguished with excellent speed and accuracy. In other terms, the generalization beyond the specific studied presentation observed here remained highly specific to the unique individual visual objects encoded. In our view, only an independent level of representation could support such a specific generalization, hence accounting for our findings. We thus believe that our results strongly make the case for the existence of a superior representational level, namely a mnemonic representation fully binding the features that characterize the object as a unique exemplar of its category for later familiarity, corresponding to the lately proposed entity level ${ }^{31}$.

The two further independent arguments pertain to the observation that viewpoint generalization specifically affected targets. First, we observed that under wakeful rest conditions, only target detection (hits rate) but not lure discrimination (false alarms rate) proved to be sensitive to perceptual distance. Second, even in the absence of wakeful rest $(\mathrm{NR})$, we observed that target detection was much less sensitive to perceptual distance than lure discrimination. We argue that the simplest explanation for the finding that a given manipulation (the availability of wakeful rest here, or the observation of a relative viewpoint generalization when no wakeful rest was available) affected so clearly targets but not lures detection, is the existence of an individual level of mnemonic representation for visual objects. Such a level of representation underwent a relative viewpoint generalization when no wakeful rest was available while rest-related consolidation achieved its full generalization across viewpoints under wakeful rest conditions.

\section{Rest-related consolidation and individual-level representations}

The present findings therefore suggest that the rest-related consolidation effects observed here on visual object recognition memory, i.e. a full viewpoint generalization but no resolution refinement, would apply to individual-level mnemonic representations, most likely nonhippocampal (due to speed constraints, see above). In contrast, as aforementioned, rest- 
related consolidation effects on visual object recognition memory most likely relying upon hippocampal mnemonic representations (i.e., no speed constraints), would refine resolution ${ }^{15}$, while full viewpoint invariance would already be reached in the absence of wakeful rest (present study, 'NRU' condition). Thus, one interesting possibility that requires further research is that rest-related consolidation would affect individual-level non-hippocampal mnemonic representations, in a different way than it would affect hippocampal mnemonic representations.

One open question relates to the neural substrates of this effect. Consolidation would involve rapid offline replay mechanisms, which are considered hippocampus-dependent ${ }^{47,48}$. Yet, the accurate fast familiarity for individual-level mnemonic representations put forward here is typically expected to be supported by the antero-lateral rhinal region (i.e. encompassing the perirhinal cortex - BA36 and BA35 - and the antero-lateral part of the entorhinal cortex $\left.{ }^{30,31}\right)$. This region, sitting at the apex of the ventral visual stream, which hierarchically represents visual objects from simple features to more and more complex conjunctions of features ${ }^{49}$, has indeed been proposed to support the entity-level (i.e. individual-level) representations of visual objects ${ }^{31}$. Hence, at least two candidate mechanisms might account for the involvement of wakeful rest-related consolidation in the viewpoint invariance of entity-level mnemonic representations. First, since nothing prevented hippocampus-dependent rest-related consolidation to occur in the present study (i.e., in SR and NR conditions), one possibility is that such consolidation would benefit the rhinal (entitylevel) mnemonic representation through hippocampal-rhinal connectivity during rest periods. Along this line, it was recently reported that the formation of non-hippocampal memories may well require sleep-related hippocampal computations ${ }^{50}$. Second, rest-related replay mechanisms have been reported outside the hippocampus $\left(e . g .{ }^{51}\right)$, and more specifically within sub hippocampal structures like the entorhinal cortex during post-encoding periods as short as 2 minutes ${ }^{52}$. Therefore, fast, spontaneous reactivation of entity-level representations during wakeful rest within sub-hippocampal, neocortical areas, might also play a role in promoting their viewpoint invariance. While these accounts are not mutually exclusive, future studies investigating within-MTL connectivity associated with rest-related consolidation of entity-level mnemonic representations would be of considerable interest.

\section{Limitations}

Our study has some limitations. First, our small sample size calls for further replication of our results. Second, given that the impact of wakeful rest varies greatly depending on the duration of the rest period, the duration of the delay phase before testing but also, and importantly, the kind of learning task involved ${ }^{10,42}$, our interpretations cannot be generalized 
beyond our specific task design. Moreover, contrary to prior reports using recognition memory tasks, objects were presented twice during the study phase. We therefore cannot rule out the contribution of an early consolidation process also taking place within the study phase. In particular, this calls for more experimental work testing whether the good performance observed even in absence of wakeful rest (NR condition) that indicates an already relative generalization, would hold under a study phase presenting objects only once. However, since the study phase was identical across experimental conditions, this does not affect our main findings on the effect of wakeful rest on individual-level mnemonic representations.

\section{Conclusion}

By investigating the role of wakeful rest upon the building of mnemonic representations for individual objects in a highly constraining familiarity-based recognition task, we have brought important and unprecedented findings calling for further research. Humans can achieve highly integrated representations of visual objects, compatible with the lately proposed 'entity level', allowing for further familiarity beyond the studied viewpoint. In turn, we unveil a role for wakeful rest-related consolidation processes in transforming these representations to make them fully immune to perceptual ambiguity, and this critically may take place within seconds postencoding. Such findings put high constraints on models of visual memory for objects, and open new avenues regarding which medial temporal lobes networks could support ultra-fast consolidation processes during awake periods of minimal sensory input, and how. 


\section{Methods}

\section{Participants}

Fifteen participants were included (all right-handed men, aged $36.9 \pm 4.4$ (range: $30-41$ )) All had normal or corrected-to-normal vision, presented with normal cognition and were free of any significant medical condition or active medication susceptible to interfere with cognition. Participants had an overall high level of education with $17.3 \pm 2.3$ years (range: 13-20) of formal education and normal levels of premorbid Full-Scale IQ as estimated with the french version of the National Adult Reading Test (f-NART ${ }^{53}$ ): $111.1 \pm 4.0$ (range: 106-121). The experimental procedures were approved by the Local Ethics Committee of the Rennes University Hospital. All participants gave their signed informed consent prior to the experiment and received financial compensation.

The order of administration remained constant across participants, who all underwent a multimodal MRI scanning session apart from the behavioral session, imaging data being not used for the present study. First, a series of questionnaires were administered to inform demographics, global health and level of education. Second, the behavioral experiment was performed. Finally, participants completed the f-NART. The whole procedure lasted around 2.5 hours.

\section{Stimuli}

Stimuli sets. Stimuli consisted of colored pictures of everyday objects cut out from their background, gathered from several stimuli databases ${ }^{32,54,55}$ (see Figure 1 for an illustration of the stimuli). A first set $(\mathrm{N}=162)$ comprised pictures of objects from 162 different subordinate categories, used for the targets (156 and 8 respectively for experimental and training blocks, see Procedure section). A second set ( $\mathrm{N}=328)$ consisted of pictures of objects grouped by pairs of distinct exemplars from 162 other different subordinate categories (156 and 8 respectively for experimental and training blocks), one exemplar being used at study, the other one serving as a lure at test (see Procedure section). Across both sets, about $27 \%$ of the objects were biological, the rest being manufactured objects. Subordinate categories were as varied as possible. Stimuli were centered on a gray background.

Stimuli manipulated parameters. Four parameters were manipulated for stimulus presentation: (1) the stimulus diagonal of the rectangle containing the stimulus (ranging from 400 to 800 pixels, for a visual angle of $4^{\circ}$ to $6^{\circ}$ ); (2) the stimulus in-plane orientation (ranging from $-40^{\circ}$ to $40^{\circ}$, with $0^{\circ}$ corresponding to upright presentation); (3) the minimum and (4) the maximum of the stimulus histogram range (i.e., the minimum and maximum luminance value of the stimulus pixels, ranging from 0 to 1 ), which allows the stimulus' contrast and luminance to be manipulated [i.e., the smaller the range is, the more contrasted the stimuli will appear 
(range width ranging from .5 to 1), while the larger the center of the range is, the more luminant the stimuli will appear (range center ranging from .25 to .75)].

Between-stimuli perceptual distances. These stimuli presentations were randomized, so that pairwise 'study-test' perceptual distances - i.e. between studied stimuli and their tested versions - presented a matched distribution between targets and lures (i.e. no difference in study-test perceptual distances between targets and lures could be evidenced using Anderson-Darling k-sample test, $p>.4$ ). Perceptual distances were computed using an euclidean distance between low- and high-level visual attributes of stimuli, derived from the HMax computational model of vision (available at http://cbcl.mit.edu/softwaredatasets/standardmodel). Briefly, following a previous study ${ }^{56}$, we captured the $\mathrm{C} 1$ responses of HMax model relating to early visual cortex (V1/V2), and the C2 responses relating to V4/posterior IT (hence respectively capturing low- and high-level visual object information ${ }^{34,57}$, on our resized images to $92 \times 92$, using the same setting (i.e. ${ }^{58}$ ) and precomputed S2 features from natural image fragments). Principal components analyses were then performed on each matrix, concatenating resp. C1 features and C2 features across all stimuli, and only the resp. 12 and 6 best components were kept and concatenated in a single matrix of 18 visual features per stimuli. The perceptual distance between two stimuli was then computed as the euclidean distance between the 18 -values vectors of each stimulus.

\section{Procedure}

Experimental setup. Participants performed the task on a MacBook Pro 13" laptop, using Matlab (Mathworks, Inc., Natick, MA) and Psychtoolbox ${ }^{59}$. Go/No-go responses were collected using a purpose-built infrared response pad, other manual responses were collected using the keyboard.

Design. The experimental session consisted of 4 different experimental conditions, each composed of 3 'study-test' blocks. These 12 'study-test' blocks were administered four-byfour, so that the first four blocks presented each of the four conditions (in a pseudo-randomized order varying across participants), the next four blocks presented each of the four conditions (in another pseudo-randomized order), etc. Before the experimental session, a training session consisted of 2 shorter blocks to ensure that the instructions were well understood; these training blocks could be repeated according to the participants preference (training blocks were 4:13 shorter than experimental blocks; they were following the 'NR'/'baseline' condition - see Conditions -, but with a -short- experimenter-paced delay phase).

Procedure. Each 'study-test' block started with a study phase in which participants had the only task of remembering the stimuli for the subsequent memory test. Each stimulus was presented 2s, separated by an inter-stimulus interval (ISI) of a duration depending on the condition (i.e. brief or long, see Condition) showing a fixation cross (a gray square of about $5^{\circ}$ 
of visual angle). The following delay phase lasted 2 min during which a task depending on the condition (i.e. navigation or cognitive rest, see Conditions) was instructed. Then, the test phase, using a response paradigm depending on the condition (i.e. speeded or unspeeded, see Conditions), assessed participants on recognition memory for individual objects, by requiring them to correctly discriminate targets from lures. Targets were 'old' stimuli encoded in the study phase but presented slightly to clearly differently (manipulating four parameters, stimuli diagonal, in-plane rotation, and minimum and maximum histogram range, see Stimuli section). Lures were 'novel' stimuli but drawn from the same subordinate categories than encoded stimuli. An experimental block consisted of 26 objects to encode across two identical presentations (with 8 to 12 intervening stimuli) during the study phase (hence consisting of 52 trials), and 13 targets (half of the encoded objects) to discriminate against 13 lures (drawn from the subordinate categories of the other half of the encoded objects) during the test phase (hence consisting of 26 trials). No stimuli (including training stimuli) were reused in any experimental block.

Conditions. Across the 4 experimental conditions ('NR', 'SR', 'DR', 'NRU') of the study-test blocks, wakeful rest was manipulated so that it was either not possible ('NR' \& 'NRU' conditions), or allowed, either for ultra-short periods during study (i.e., longer ISIs, 'SR') or during the two minutes of the delay phase. Figure $1 \mathrm{C}$ presents the chosen manipulation for each phase in the different conditions. In the study phase, a long ISI duration pseudorandomized between 2000 to 3000 ms was used in the 'SR' condition to serve as an ultrashort period of cognitive rest (see Introduction), while a short ISI duration pseudo-randomized between 100 to 200 ms was used in the three other conditions, avoiding any wakeful rest during the study phase. In the delay phase, participants were asked to undergo 2 min of wakeful rest in the 'DR' condition, thus allowing the opportunity of rest-related consolidation, while they were asked to perform a 2-min mental navigation task in the three other conditions, preventing any cognitive rest (see Introduction). Wakeful rest instructions were to wait in silence for $2 \mathrm{~min}$, without closing eyes nor focusing on any specific thoughts, but more letting the mind flow, in a general relaxing attitude. A short interview after each wakeful rest period ensured that no participant actively rehearsed the stimuli during rest. In the mental navigation task, participants were instructed to describe within 2 min to the experimenter as detailed as possible personally well-known commute routes while vividly visualizing it; 9 different routes were pre-experimentally prepared, randomly chosen by the experimenter (e.g. route from home to bakery, route from work to home, route from childhood home to school, etc.), and adapted or lengthen when necessary. In the test phase, recognition memory was assessed using an unspeeded Yes/No response paradigm in the 'NRU' condition, theoretically letting familiarity and recollection processes contributing performance (see Introduction), while it was assessed in the three other conditions using a speeded Go/No-Go paradigm that greatly limit 
the possibility of using recollection-based retrieval strategies (see Introduction). The unspeeded Yes/No response paradigm presented the stimulus until a Yes- or a No- response was given (using resp. keys ' $\mathrm{O}$ ' and ' $\mathrm{N}$ '); then a confidence questionnaire (3-level Likert scale) was supplementarily proposed asking participants' to indicate using the digit keys between 1 and 3 'With which confidence did you just give your response? 1 - not sure at all ; 2 - rather uncertain ; 3 - certain'. The speeded Go/No-Go paradigm implemented the Speed and Accuracy Boosting (SAB) procedure (e.g. Besson et al., 2012, 2017) using a 600-ms response deadline until which stimuli were presented and giving an auditory feedback ('correct' or 'incorrect') at the response offset (i.e. at the Go-response, or at the response deadline if no Go-response was given) (for a more detailed description of the SAB, see e.g. ${ }^{32,60}$ ); participants were instructed to give their Go-response as fast as they could during the stimulus presentation (i.e. before reaching the response deadline). In both unspeeded and speeded test paradigms, ISI durations were pseudo-randomized between 1800 ms and 2400 ms.

\section{Statistical analyses}

Data and statistical analyses were performed with Matlab. We implemented statistical analyses of the effect of the conditions at the participant-level using generalized linear mixed model (GLMM; with the 'fitglme' MATLAB function) including a random intercept for participants (model: 'score condition + (condition | participant)'; akin to repeated-measures ANOVAs) and using a 'normal' distribution. Interested to estimate the effect of each condition compared to the 'NR' condition, we chose a 'reference' coding (referenced on this condition), and explored the general main effect of the conditions as well as each fixed effect of the three other conditions with respect to the 'NR' condition. Scores of interest regarding recognition memory performance were computed within the signal detection theory framework. Apart from Hits (correct Go responses) and False alarms (incorrect Go responses) rates and following ${ }^{61}$ we computed $\mathrm{Az}$ to estimate accuracy, namely, how well participants discriminated between targets and distractors, and B", a nonparametric metric of bias (using a default value for the variance ratio of $1^{62}$ ). We preferred these indices to the parametric $d$ ' and $C$ indices of accuracy and bias, as they are more robust to underlying assumptions about response distributions ${ }^{61,63}$. The corresponding formulae were implemented using a dedicated Excel workbook (Gaetano, 2017; Signal detection theory calculator 1.2 [Excel workbook downloaded from https://www.researchgate.net/profile/Justin_Gaetano2]). To assess the effect of the studytest perceptual distances on responses, we also extracted the beta-values estimated for the effect of the perceptual distance at the trial-level from a GLMM (model: 'resp dist', distribution: 'binomial', link function: 'logit') assessed independently on targets and lures for each participant and each condition, adding two supplementary scores of interest: hits and false alarms sensitivity to study-test perceptual changes. In order to assess for each condition 
whether these two scores were significant (i.e. significantly different from 0 ) we performed ttests against 0 . For all analyses, the statistical threshold was set at $p<.05$. 


\section{References}

1. Standing, L. Learning 10,000 pictures. Q. J. Exp. Psychol. 25, 207-222 (1973).

2. Brady, T. F., Konkle, T., Alvarez, G. A. \& Oliva, A. Visual long-term memory has a massive storage capacity for object details. Proc. Natl. Acad. Sci. 105, 14325-14329 (2008).

3. DiCarlo, J. J. \& Cox, D. D. Untangling invariant object recognition. Trends Cogn. Sci. 11, 333-341 (2007).

4. Thorpe, S., Fize, D. \& Marlot, C. Speed of processing in the human visual system. Nature 381, 520-522 (1996).

5. Brown, M. W. \& Aggleton, J. P. Recognition memory: what are the roles of the perirhinal cortex and hippocampus? Nat. Rev. Neurosci. 2, 51-61 (2001).

6. Hintzman, D. L., Caulton, D. A. \& Levitin, D. J. Retrieval dynamics in recognition and list discrimination: Further evidence of separate processes of familiarity and recall. Mem. Cogn. 26, 449-462 (1998).

7. McElree, B., Dolan, P. O. \& Jacoby, L. L. Isolating the Contributions of Familiarity and Source Information to Item Recognition: A Time Course Analysis. J. Exp. Psychol. Learn. Mem. Cogn. 25, 563-582 (1999).

8. Dudai, Y., Karni, A. \& Born, J. The Consolidation and Transformation of Memory. Neuron 88, 20-32 (2015).

9. Stickgold, R. Sleep-dependent memory consolidation. Nature 437, 1272-1278 (2005).

10. Wamsley, E. J. Memory Consolidation during Waking Rest. Trends Cogn. Sci. 23, 171-173 (2019).

11. Wamsley, E. J. \& Summer, T. Spontaneous Entry into an "Offline " State during Wakefulness : A Mechanism of Memory Consolidation? J. Cogn. Neurosci. 1-21 (2020).

12. Mortamais, M. et al. Detecting cognitive changes in preclinical Alzheimer's disease: A review of its feasibility. Alzheimer's Dement. 13, 468-492 (2017).

13. Craig, M., Dewar, M., Della Sala, S. \& Wolbers, T. Rest boosts the long-term retention of new spatial memories. Hippocampus 25, 1017-1027 (2015).

14. Craig, M., Dewar, M., Harris, M. A., Della Sala, S. \& Wolbers, T. Wakeful rest promotes the integration of spatial memories into accurate cognitive maps. Hippocampus 26, 185-193 (2016).

15. Craig, M. \& Dewar, M. Rest-related consolidation protects the fine detail of new memories. Sci. Rep. 8, 1-9 (2018).

16. Dewar, M., Alber, J., Butler, C., Cowan, N. \& Della Sala, S. Brief Wakeful Resting Boosts New Memories Over the Long Term. Psychol. Sci. 23, 955-960 (2012). 
17. Dewar, M., Alber, J., Cowan, N. \& Sala, S. Della. Boosting long-term memory via wakeful rest: Intentional rehearsal is not necessary, consolidation is sufficient. PLoS One 9, (2014).

18. Sacripante, R., Mclntosh, R. D. \& Della Sala, S. Benefit of wakeful resting on gist and peripheral memory retrieval in healthy younger and older adults. Neurosci. Lett. 705 , 27-32 (2019).

19. Martini, M., Zamarian, L., Sachse, P., Martini, C. \& Delazer, M. Wakeful resting and memory retention: a study with healthy older and younger adults. Cogn. Process. 20, 125-131 (2019).

20. Martini, M., Martini, C., Maran, T. \& Sachse, P. Effects of post-encoding wakeful rest and study time on long-term memory performance. J. Cogn. Psychol. 30, 558-569 (2018).

21. Mercer, T. Wakeful rest alleviates interference-based forgetting. Memory 23, 127-137 (2015).

22. Moscovitch, M., Cabeza, R., Winocur, G. \& Nadel, L. Episodic memory and beyond: The hippocampus and neocortex in transformation. Annu. Rev. Psychol. 67, 105-134 (2016).

23. Eichenbaum, H., Sauvage, M., Fortin, N., Komorowski, R. \& Lipton, P. Neuroscience and Biobehavioral Reviews Towards a functional organization of episodic memory in the medial temporal lobe. 36, 1597-1608 (2012).

24. Yonelinas, A. P. The Nature of Recollection and Familiarity: A Review of 30 Years of Research. J. Mem. Lang. 46, 441-517 (2002).

25. Ranganath, C. \& Ritchey, M. Two cortical systems for memory-guided behaviour. Nat. Rev. Neurosci. 13, 713-726 (2012).

26. Whittlesea, B. W. A. \& Williams, L. D. The Discrepancy-Attribution Hypothesis: I. The Heurisrtic Basis of Feelings of Familiarity. J. Exp. Psychol. Learn. Mem. Cogn. 27, 313 (2001).

27. Lucas, H. D., Taylor, J. R., Henson, R. N. \& Paller, K. A. Many roads lead to recognition: Electrophysiological correlates of familiarity derived from short-term masked repetition priming. Neuropsychologia 50, 3041-3052 (2012).

28. Oppenheimer, D. M. The secret life of fluency. Trends Cogn. Sci. 12, 237-241 (2008).

29. Erez, J., Cusack, R., Kendall, W. \& Barense, M. D. Conjunctive Coding of Complex Object Features. Cereb. Cortex 26, 2271-2282 (2016).

30. Besson, G., Simon, J., Salmon, E. \& Bastin, C. Familiarity for entities as a sensitive marker of antero-lateral entorhinal atrophy in amnestic mild cognitive impairment. Cortex 128, 61-72 (2020).

31. Bastin, C. et al. An Integrative Memory model of recollection and familiarity to 
understand memory deficits. Behav. Brain Sci. (2019).

doi:10.1017/S0140525X19000621

32. Besson, G., Ceccaldi, M., Didic, M. \& Barbeau, E. J. The speed of visual recognition memory. Vis. cogn. 20, 1131-1152 (2012).

33. Besson, G. et al. Fast, but not slow, familiarity is preserved in patients with amnestic mild cognitive impairment. Cortex 65, 36-49 (2015).

34. Riesenhuber, M. \& Poggio, T. Hierarchical models of object recognition in cortex. Nat. Neurosci. 2, 1019-1025 (1999).

35. Craig, M., Ottaway, G. \& Dewar, M. Rest on it: Awake quiescence facilitates insight. Cortex 109, 205-214 (2018).

36. Graveline, Y. M. \& Wamsley, E. J. The impact of sleep on novel concept learning. Neurobiol. Learn. Mem. 141, 19-26 (2017).

37. Borota, D. et al. Post-study caffeine administration enhances memory consolidation in humans. Nat. Neurosci. 17, 201-203 (2014).

38. Doxey, C. R., Hodges, C. B., Bodily, T. A., Muncy, N. M. \& Kirwan, C. B. The effects of sleep on the neural correlates of pattern separation. Hippocampus 28, 108-120 (2018).

39. Ben-Yakov, A., Eshel, N. \& Dudai, Y. Hippocampal immediate poststimulus activity in the encoding of consecutive naturalistic episodes. J. Exp. Psychol. Gen. 142, 12551263 (2013).

40. Jonin, P.-Y. et al. Superior explicit memory despite severe developmental amnesia: In-depth case study and neural correlates. Hippocampus 1-19 (2018). doi:10.1002/hipo.23010

41. Despouy, E. et al. A Fast Visual Recognition Memory System in Humans Identified Using Intracerebral ERP. Cereb. Cortex 30, 2961-2971 (2020).

42. Tucker, M. A., Humiston, G. B., Summer, T. \& Wamsley, E. Comparing the effects of sleep and rest on memory consolidation. Nat. Sci. Sleep 12, 79-91 (2020).

43. Payne, J. D., Stickgold, R., Swanberg, K. \& Kensinger, E. A. Sleep preferentially enhances memory for emotional components of scenes. Psychol. Sci. 19, 781-788 (2008).

44. Utochkin, I. S. \& Brady, T. F. Independent Storage of Different Features of Real-World Objects in Long-Term Memory. J. Exp. Psychol. Gen. 1-55 (2019). doi:10.1037/xge0000664

45. Brady, T. F., Konkle, T., Alvarez, G. A. \& Oliva, A. Real-world objects are not represented as bound units: Independent forgetting of different object details from visual memory. J. Exp. Psychol. Gen. 142, 791-808 (2013).

46. Brady, T. F., Konkle, T. \& Alvarez, G. A. A review of visual memory capacity: Beyond 
individual items and toward structured representations. J. Vis. 11, 1-34 (2011).

47. Carr, M. F., Jadhav, S. P. \& Frank, L. M. Hippocampal replay in the awake state: A potential substrate for memory consolidation and retrieval. Nat. Neurosci. 14, 147153 (2011).

48. Foster, D. J. \& Wilson, M. A. Reverse replay of behavioural sequences in hippocampal place cells during the awake state. Nature 440, 680-683 (2006).

49. Saksida, L. M. \& Bussey, T. J. The representational-hierarchical view of amnesia: Translation from animal to human. Neuropsychologia 48, 2370-2384 (2010).

50. Sawangjit, A. et al. The hippocampus is crucial for forming non-hippocampal longterm memory during sleep. Nature 564, 109-113 (2018).

51. Hoffman, K. L. \& McNaughton, B. L. Coordinated reactivation of distributed memory traces in primate neocortex. Science (80-. ). 297, 2070-2073 (2002).

52. Staresina, B. P., Alink, A., Kriegeskorte, N. \& Henson, R. N. Awake reactivation predicts memory in humans. Proc. Natl. Acad. Sci. U. S. A. 110, 21159-21164 (2013).

53. Mackinnon, A. \& Mulligan, R. Estimation de l'intelligence prémorbide chez les francophones. Encephale. 31, 31-43 (2005).

54. Brodeur, M. B., Dionne-Dostie, E., Montreuil, T. \& Lepage, M. The bank of standardized stimuli (BOSS), a new set of 480 normative photos of objects to be used as visual stimuli in cognitive research. PLoS One 5, (2010).

55. Kovalenko, L. Y., Chaumon, M. \& Busch, N. A. A pool of pairs of related objects (POPORO) for investigating visual semantic integration: Behavioral and electrophysiological validation. Brain Topogr. 25, 272-284 (2012).

56. Clarke, A., Devereux, B. J., Randall, B. \& Tyler, L. K. Predicting the time course of individual objects with MEG. Cereb. Cortex 25, 3602-3612 (2015).

57. Serre, T., Oliva, A. \& Poggio, T. A feedforward architecture accounts for rapid categorization. Proc. Natl. Acad. Sci. 104, 6424-6429 (2007).

58. Serre, T., Wolf, L. \& Poggio, T. Object recognition with features inspired by visual cortex. Proc. - 2005 IEEE Comput. Soc. Conf. Comput. Vis. Pattern Recognition, CVPR 2005 II, 994-1000 (2005).

59. Brainard, D. H. The Psychophsycis Toolbox. Spat. Vis. 10, 433-436 (1997).

60. Besson, G. et al. From face processing to face recognition: Comparing three different processing levels. Cognition 158, 33-43 (2017).

61. Verde, M. F., Macmillan, N. A. \& Rotello, C. M. Measures of sensitivity based on a single hit rate and false alarm rate: The accuracy, precision, and robustness of',A z, andA'. Percept. Psychophys. 68, 643-654 (2006).

62. Grier, J. B. Nonparametric indexes for sensitivity and bias: computing formulas. Psychol. Bull. 75, 424-429 (1971). 
63. Stanislaw, H. \& Todorov, N. Calculation of signal detection theory measures. Behav. Res. Methods, Instruments, Comput. 31, 137-149 (1999). 